\title{
The Comparison Between Molecular Tumour Profiling in Microdissected and Surgical Tissue Samples
}

\author{
IZABELA LACZMANSKA $^{1}$, MARIA SASIADEK ${ }^{1}$ and LUKASZ LACZMANSKI ${ }^{2}$ \\ ${ }^{1}$ Genetics Department, Wroclaw Medical University, Wroclaw, Poland; \\ ${ }^{2}$ Hirszfeld Institute of Immunology and Experimental Therapy, Polish Academy of Sciences, Wroclaw, Poland
}

\begin{abstract}
Background/Aim: Laser capture microdissection $(L C M)$ is one of the most important tools in molecular and histopathological tissue analysis. We compared the expression level of protein phosphatase genes in LCM and surgical colorectal cancer samples to evaluate whether there is a significant difference in molecular profiling. Materials and Methods: The expression levels of 99 protein phosphatase and 15 control genes were analysed in 104 microdissected, 81 surgical colorectal cancer and 25 control samples. Microarray expression data were obtained from the GEO Database of the National Center for Biotechnology Information. Results: The analysis revealed that over $60 \%$ of expression results were in agreement with LCM and surgically obtained samples while $32 \%$ of non-matched results belonged to the group where no effect was observed in LCM samples and down-regulation-or overexpression was reported in surgical samples. Conclusion: Generally, it is more likely to find critical genetic alterations in surgically obtained than in LCM samples.
\end{abstract}

Nowadays molecular analysis of cancer tissues at the genomic, transcriptomic or epigenetic level is a standard procedure both in scientific and diagnostic fields. Proper histopathological analysis, especially immunodiagnostics, can inform about the classification, stage of the tumour, its progression or a possible response to an applied therapy (1). However, the percentage of cancer cells in examined samples or the percentages of different cancer cell subpopulations, e.g. representing different stages, may greatly differ in two tissue samples obtained even in the close vicinity. Because of cancer tissue heterogeneity and the presence of different

Correspondence to: Izabela Laczmanska, Department of Genetics, Medical University, Marcinkowskiego 1, 50-368 Wroclaw, Poland. Tel: +48 0717841258, Fax: +48 0717840063, e-mail: izabela.laczmanska@ umed.wroc.pl

Key Words: Microdissection, LCM, PTPs, expression colorectal cancer. types of cancer and non-cancer cells in one sample obtained during surgery, the analysis of the molecular events characteristic for cancer biology may be problematic and the way of sampling may greatly influence the results of all molecular analyses (2-4). Therefore, techniques enabling better separation of tissue samples and enrichment of a specific cell type within a surgically- or biopsy- obtained tissue are widely applied either in research studies or in diagnostics (3). Laser capture microdissection (LCM), used since 1996, is one of the most important tools in tissue analysis in histopathology and molecular findings (3). An undoubted advantage of this technique is the reduction of the amount of the collected material and the improvement of its quality. Many studies on genomic DNA, RNA and proteins isolated from laser-capture tissue samples have been described recently (5). The number and diversity of these studies have steadily increased, particularly because of the introduction of precise medicine in cancer therapy and treatment. Nowadays molecular profiling in cancer allows one to choose tailored therapy that significantly increases the chance of survival and constitutes one of the bases of precision oncology. In comparison to surgically obtained samples, LCM focuses on specific cells and gives the possibility to examine one cell subpopulation $(3,5)$.

Effectiveness of targeted therapies in colorectal cancer depends on the proper selection of patients according to the genetic status of crucial genes such as for anti-EGFR therapy, where $B R A F \mathrm{~V} 600 \mathrm{E}$ and $K R A S$ codons 12 and 13 in exon 2 mutations and also NRAS and PIK3CA mutations are strong predictors of resistance to this therapy and correlate with a lower response rate (6). Because of the high costs and toxicity of such therapies, the molecular information obtained from cancer tissues is crucial for the patient; therefore the proper way of obtaining samples is pivotal (7).

Recently many opportunities have arisen from liquid biopsy and droplet digital PCR technology. Circulating tumour cells or cell-free circulating tumour DNA (cfDNA) have been shown to be strong prognostic factors but they are still not approved in daily clinical practice (7). Therefore, surgically- and LCM- obtained samples are still a standard 
material for molecular profiling (7). Moreover, beside basic mutation analysis, molecular profiling of different groups of genes in cancers is one of the principal subjects of basic research (8).

Sporadic colorectal cancer (CRC) is one of the most common cancers in the world and one of the most frequent cancer-related causes of death. Genetic alterations together with environmental factors such as diet and lifestyle play a crucial role in CRC pathogenesis (8). Among the most examined groups of genes in CRC, tyrosine kinases and tyrosine phosphatases play major roles $(2,9-11)$. Recent articles strongly suggest not only the suppressive, but also the oncogenic role of protein tyrosine phosphatases (PTPs) in cancer development and progression $(10,12-15)$. The crucial role of protein tyrosine phosphatases is reflected by the rising number of publications about this class of genes in different cancers. Various alterations in PTP genes, such as gene deletions and duplications, point mutations and epigenetic modifications, and also changes in their expression levels, have been reported among others in prostate, breast, colon, lung, liver, pancreas and thyroid cancer (10).

In our study we focused on microarray expression of 114 genes, including 99 protein tyrosine phosphatases and 15 control genes in LCM versus surgically obtained colorectal cancer samples, to evaluate whether molecular profiling in microdissected tumour samples corresponds to surgical tissue samples.

\section{Materials and Methods}

We analysed data for the expression level of 99 protein phosphatase genes placed in Hugo Gene Nomenclature Committee (HGNC) Website, access date 07-06-2017, and 15 control genes described as important for sporadic colorectal cancer (16-18). Microarray expression data were obtained from GEO Database of the National Center for Biotechnology Information (NCBI) (GSE21510 (19), GSE35896 (20)). mRNA was isolated from LCM samples of 104 colorectal patients, 81 homogenized tissues of colorectal patients and 25 homogenized normal tissues. Colorectal tumour samples were hybridized to Affymetrix HGU133 Plus 2.0 expression arrays (GPL570). Datasets were normalized by the robust multi-array average (RMA) method. Data were presented as $\log 2$-transformed values by RMA. Log2 fold change (log2FC) was calculated as the difference between two averages: the $\log 2$ RMA signal obtained from cancer tissue and the $\log 2$ RMA signal obtained from normal tissue. A $\log 2 \mathrm{FC}$ value below -1 was described as down expression and $\log 2 \mathrm{FC}>1$ was described as overexpression.

\section{Results}

The analysis of 15 control genes in 104 LCM samples and 81 surgical samples revealed the same expression levels for 14 genes $(93 \%)$ and different results for 1 gene $(7 \%)$ (Table I). For 11 matched genes (73\%) lower expression either in LCM or in surgical samples was observed. Only 2 genes
Table I. Comparison between expression levels of selected control genes in LCM samples versus surgical samples.

\begin{tabular}{lcc}
\hline LCM/SURGERY & List & No. \\
\hline Matched & TGFBI, TCN1 & $2(13 \%)$ \\
Over & $A B C G 2$, AQP8, SPIB, CA7, & $11(73 \%)$ \\
Down & $C L D N 8$, SCNN1B, SLC30A10, \\
\multicolumn{3}{c}{ CD177, PADI2, CWH43 } \\
No effect & IL6R & $1(7 \%)$ \\
All matched & & $14(93 \%)$ \\
Not matched & --- & \\
Over/no effect & -- & $1(7 \%)$ \\
Down/no effect & SPP1 \\
Over/ down & --- & \\
Down/over & --- & $1(7 \%)$ \\
No effect/over & -- & $15(100 \%)$ \\
No effect/down & & \\
ALL not matched & & \\
ALL & &
\end{tabular}

showed higher expression and 1 gene showed normal expression in both types of samples. One gene with different expression was down-regulated in surgical samples and overexpressed in LCM samples (Table I).

The analysis of 99 PTP genes in 104 LCM samples and 81 surgical samples revealed the same expression results for 60 genes $(61 \%)$ and different results for 39 genes (39\%) (Table II). For 46 genes (47\%) no changes in expression level either in LCM or in surgical samples were observed. Only 7 genes showed higher expression and 7 genes showed lower expression in both types of samples.

Among genes with different expression results for LCM and surgical samples, 32 genes with no changes in LCM samples showed overexpression (14 genes) or downregulation (18 genes) in surgical samples. For one gene (PTPN12) and two genes (ACP2 and SSU72) which were over- and down-regulated respectively in LCM samples no change in gene expression level in surgically obtained samples was found. Conversely, for 3 genes that were overexpressed in LCM samples we observed downregulation in surgical samples and for one gene (PHPTl) we observed down-regulation in LCM and overexpression in surgically obtained samples (Table II).

\section{Discussion}

Precise and accurate analysis of gene mutational or expression status is one of the most important steps in pharmacogenetics and tailored therapy (21). Obtaining the appropriate sample may not only have a strong influence on 
Table II. Comparison between expression level of selected PTP genes in LCM samples versus surgical samples.

\begin{tabular}{|c|c|c|}
\hline LCM/surgery & Genes & No. \\
\hline \multicolumn{3}{|l|}{ Matched } \\
\hline Over & ACP6, CDC25B, CDKN3, EPM2AIP1, DUSP10, DUSP14, DUSP4 & $7(7 \%)$ \\
\hline Down & DUSP1, DUSP5, PDXP, PTPRF, PTPRH, PTPRR, TNS1 & $7(7 \%)$ \\
\hline No effect & $\begin{array}{l}\text { PTPDC1, PTPMT1, PTPN14, PTPN18, PTPN22, PTPN23, PTPN3, PTPN5, PTPN6, PTPN9, } \\
\text { PTPRB, PTPRC, PTPRD, PTPRE, PTPRG, PTPRJ, PTPRK, PTPRM, PTPRS, PTPRT, PTPRU, } \\
\text { PTPRZ1, CDC14C, CDC14A, EPM2A, SSH1, SSH2, UBASH3B, DUSP11, DUSP12, DUSP16, } \\
\text { DUSP18, DUSP19, DUSP2, DUSP22, DUSP22/LOC100653247, DUSP23, DUSP28, DUSP5P1, } \\
\text { DUSP6, MDP1, PGAM5, PTEN/PTENP1, TNS2, TPTE, TPTE2 } \\
\text { All matched }\end{array}$ & $46(47 \%)$ \\
\hline \multicolumn{3}{|l|}{ Not matched } \\
\hline Over/no effect & PTPN12 & $1(1 \%)$ \\
\hline Down/no effect & $A C P 2, S S U 72$, & $2(2 \%)$ \\
\hline Over/ down & $A C P 1, P T P N 11, P T P N 2$ & $3(3 \%)$ \\
\hline Down/over & PHPT1 & $1(1 \%)$ \\
\hline No effect/over & $\begin{array}{l}\text { ACP5, CDC14B, CDC25C, DUSP27, DUSP3, PTP4A3, PTPN1, PTPN13, PTPN4, PTPRA, PTPRN2, } \\
\text { RNGTT, STYX, STYXL1 }\end{array}$ & $14(14 \%)$ \\
\hline No effect/down & $\begin{array}{l}\text { CDC25A, DUSP13, DUSP15, DUSP21, DUSP26, DUSP8/LOC101927562, DUSP9, PTEN, PTP4A1, } \\
\text { PTP4A2, PTPN20B, PTPN21, PTPN7, PTPRN, PTPRO, SSH3, SSU72P8, SSUH2 }\end{array}$ & $18(18 \%)$ \\
\hline All not matched & & $39(39 \%)$ \\
\hline ALL & & $99(100 \%)$ \\
\hline
\end{tabular}

the result of molecular analysis but also be a cause of false negative results. Because of unquestionable accuracy of LCM technique and strong heterogeneity of colorectal cancer we assumed that the discrepancy between results for LCM and surgically obtained samples would be relevant. To validate our results of the phosphatase genes we also analysed 15 control genes, previously described as good indicators of the candidate genes that correlate with CRC. According to two previous published meta-analyses of the expression profiling of colorectal tumours we chose as control genes: $A B C G 2, A Q P 8, S P I B, C A 7, C L D N 8$, SCNN1B, SLC30A10, CD177, PADI2, TGFBI, GUCA2B, IL6R, SPP1, TCN1 and CWH43 (AQP8, SPIB, CA7 were overlapping in both articles). Most of these genes were reported to be down-regulated in colorectal cancer tissue except SPP1, TCN1 and TGFBI, for which overexpression was reported $(16,17)$.

The analysis of control genes revealed that either in LCM or in surgically obtained samples 14 out of 15 genes presented the same level of expression; the exception was $S P P 1$, which was overexpressed in LCM and down-regulated in surgically obtained samples. In previously described wide analyses SPP1 was shown to be overexpressed in CRC tissues; therefore, the results obtained from LCM samples seem to be relevant. For all control genes except ILR6 we obtained the same results as in previously reported analyses $(16,17)$. These results suggest that there is no difference in expression of genes that strongly correlate with CRC in LCM and surgically obtained samples.

Our analysis of phosphatase genes revealed that over $60 \%$ of expression results were in agreement with LCM and surgically obtained samples. Thirty-two phosphatase genes (32\%) of non-matched results belonged to the group where no effect was observed in LCM samples while in surgical samples down-regulation- or overexpression was reported (14 and $18 \%$ respectively). This suggests that despite the accuracy of LCM technique a huge number of molecular events present in an examined cancer tissue may be overlooked in microdissected samples. Moreover, the lack of precision in microscale may paradoxically guarantee more relevant results with higher specificity especially for genes that are not strongly correlated with CRC.

For a heterogeneous cancer like colorectal cancer a standard histopathological analysis for distinction of cancer and non-cancer cells seems to be sufficient to choose a representative sample for molecular analyses. Despite the risk that some genetic alterations present in a small number of cells may not be detected in surgically obtained samples, generally it is more likely to find critical genetic alterations in tissues sampled in this way than in those sampled locally with high precision and accuracy using LCM. Moreover, the sensitivity of currently applied molecular techniques allows one to detect genetic alterations present even only in a small percentage of cells in an examined tissue. 
Protein tyrosine phosphatases are reported to act either as suppressor genes or as oncogenes $(10,12,13)$. This double role arises from the importance of the reaction they catalyze - dephosphorylation of many proteins and therefore the change of their activity in different crucial biological pathways (12). Our analysis revealed that in surgicallyobtained tissue $22 \%$ of phosphatase genes were overexpressed while $28 \%$ were down-regulated. For $50 \%$ of all known phosphatase genes there is a significant change in expression in colorectal cancer tissue versus healthy tissue. This is another result confirming the high impact of this class of genes on CRC development and progression.

\section{Acknowledgements}

Publication supported by Wroclaw Centre of Biotechnology, programme The Leading National Research Centre (KNOW) for years 2014-2018.

\section{References}

1 Kourea $\mathrm{H}$ and Kotoula V: Towards tumor immunodiagnostics. Ann Transl Med 4: 263-263, 2016.

2 Schmidt F and Efferth T: Tumor Heterogeneity, Single-Cell Sequencing, and Drug Resistance. Pharmaceuticals 9: 33, 2016.

3 Liu H, McDowell TL, Hanson NE, Tang X, Fujimoto J and Rodriguez-Canales $\mathrm{J}$ : Laser capture microdissection for the investigative pathologist. Vet Pathol 51: 257-269, 2014.

4 Datta S, Malhotra L, Dickerson R, Chaffee S, Sen CK and Roy S: Laser capture microdissection: Big data from small samples. Histol Histopathol 30: 1255-1269, 2015.

5 Legres LG, Janin A, Masselon C and Bertheau P: Beyond laser microdissection technology: follow the yellow brick road for cancer research. Am J Cancer Res 4: 1-28, 2014.

6 Tran NH, Cavalcante LL, Lubner SJ, Mulkerin DL, LoConte NK, Clipson L, Matkowskyj KA and Deming DA: Precision medicine in colorectal cancer: the molecular profile alters treatment strategies. Ther Adv Med Oncol 7: 252-262, 2015.

7 Cabel L, Proudhon C, Gortais H, Loirat D, Coussy F, Pierga J$\mathrm{Y}$ and Bidard F-C: Circulating tumor cells: clinical validity and utility. Int J Clin Oncol: 1-10, 2017.

8 Mármol I, Sánchez-de-Diego C, Pradilla Dieste A, Cerrada E and Rodriguez Yoldi MJ: Colorectal Carcinoma: A General Overview and Future Perspectives in Colorectal Cancer. Int J Mol Sci 18, 2017. doi: 10.3390/ijms18010197. [Epub ahead of print]

9 Hoekstra E, Peppelenbosch MP and Fuhler GM: The role of protein tyrosine phosphatases in colorectal cancer. Biochim Biophys Acta - Rev Cancer 1826: 179-188, 2012.

10 Bollu LR, Mazumdar A, Savage MI and Brown PH: Molecular Pathways: Targeting Protein Tyrosine Phosphatases in Cancer. Clin Cancer Res 23: 2136-2142, 2017.
$11 \mathrm{He} \mathrm{R}$, Yu Z, Zhang $\mathrm{R}$ and Zhang Z: Protein tyrosine phosphatases as potential therapeutic targets. Acta Pharmacol Sin 35: 1227-1246, 2014.

12 Zhao S, Sedwick D and Wang Z: Genetic alterations of protein tyrosine phosphatases in human cancers. Oncogene 34: 38853894, 2015.

13 Laczmanska I, Karpinski P, Kozlowska J, Bebenek M, Ramsey D, Sedziak T, Ziolkowski P and Sasiadek MM: Copy number alterations of chromosomal regions enclosing protein tyrosine phosphatase receptor-like genes in colorectal cancer. Pathol Res Pract 210: 893-896, 2014.

14 Laczmanska I, Skiba P, Karpinski P, Bebenek M and Sasiadek MM: Customized array comparative genomic hybridization analysis of 25 phosphatase-encoding genes in colorectal cancer tissues. Cancer Genomics Proteomics 14: 69-74, 2017.

15 Laczmanska I, Karpinski P, Gil J, Laczmanski L, Bebenek M and Sasiadek MM: High PTPRQ expression and its relationship to expression of PTPRZ1 and the presence of KRAS mutations in colorectal cancer tissues. Anticancer Res 36: 677-681, 2016.

16 Shangkuan W-C, Lin H-C, Chang Y-T, Jian C-E, Fan H-C, Chen K-H, Liu Y-F, Hsu H-M, Chou H-L, Yao C-T, Chu C-M, Su S$\mathrm{L}$ and Chang C-W: Risk analysis of colorectal cancer incidence by gene expression analysis. PeerJ 5: e3003, 2017.

17 Chu C-M, Yao C-T, Chang Y-T, Chou H-L, Chou Y-C, Chen KH, Terng H-J, Huang C-S, Lee C-C, Su S-L, Liu Y-C, Lin F-G, Wetter $\mathrm{T}$ and Chang $\mathrm{C}-\mathrm{W}$ : Gene expression profiling of colorectal tumors and normal mucosa by microarrays metaanalysis using prediction analysis of microarray, artificial neural network, classification, and regression trees. Dis Markers 2014: 1-11, 2014.

18 Gray KA, Yates B, Seal RL, Wright MW and Bruford EA: Genenames.org: The HGNC resources in 2015. Nucleic Acids Res 43: D1079-D1085, 2015.

19 Tsukamoto S, Ishikawa T, Iida S, Ishiguro M, Mogushi K, Mizushima H, Uetake H, Tanaka H and Sugihara K: Clinical significance of osteoprotegerin expression in human colorectal cancer. Clin Cancer Res 17: 2444-2450, 2011.

20 Schlicker A, Beran G, Chresta CM, McWalter G, Pritchard A, Weston S, Runswick S, Davenport S, Heathcote K, Castro DA, Orphanides G, French T and Wessels LF: Subtypes of primary colorectal tumors correlate with response to targeted treatment in colorectal cell lines. BMC Med Genomics 5: 66, 2012.

$21 \mathrm{Lu}$ D-Y, Lu T-R, Xu B and Ding J: Pharmacogenetics of cancer therapy: breakthroughs from beyond? Futur Sci OA 1: fso.15.80, 2015. 